\title{
Anti-adult T-cell leukemia/lymphoma activity of cerdulatinib, a dual SYK/JAK kinase inhibitor
}

\author{
CHIE ISHIKAWA $^{1,2}$, MASACHIKA SENBA ${ }^{3}$ and NAOKI MORI ${ }^{1}$ \\ ${ }^{1}$ Department of Microbiology and Oncology, Graduate School of Medicine, University of The Ryukyus, Nishihara, \\ Okinawa 903-0215; ${ }^{2}$ Division of Health Sciences, Transdisciplinary Research Organization for Subtropics \\ and Island Studies, University of The Ryukyus, Nishihara, Okinawa 903-0213; ${ }^{3}$ Department of Pathology, \\ Institute of Tropical Medicine, Nagasaki University, Nagasaki 852-8523, Japan
}

Received March 9, 2018; Accepted July 3, 2018

DOI: $10.3892 /$ ijo.2018.4513

\begin{abstract}
Adult T-cell leukemia/lymphoma (ATLL) constitutes an aggressive malignancy caused by human T-cell leukemia virus type 1 (HTLV-1) that is resistant to available chemotherapeutics. The constitutive activation of Janus kinase (JAK)-signal transducer and activator of transcription (STAT) signaling is an important feature of ATLL, and spleen tyrosine kinase (SYK) is overexpressed in HTLV-1transformed T-cell lines. In this study, we evaluated the effects of SYK- (PRT060318) or JAK- (JAK inhibitor 1) selective inhibitors and the dual SYK/JAK inhibitor, cerdulatinib, on the viability of HTLV-1-transformed and ATLL-derived T-cell lines. Cell proliferation, viability, cell cycle, apoptosis and intracellular signaling cascades were analyzed by the watersoluble tetrazolium-8 assay, flow cytometry and western blot analysis. HTLV-1-infected T-cell lines were sensitive to both SYK-selective and pan-JAK inhibitors, whereas cerdulatinib more potently suppressed cell proliferation and reduced cell viability than either of these agents alone. By contrast, the cytotoxic effects of cerdulatinib on uninfected T-cell lines and peripheral blood mononuclear cells from a healthy donor were less pronounced. Cerdulatinib induced cell cycle arrest in the $\mathrm{G}_{2} / \mathrm{M}$ phase, which was associated with a decreased cyclindependent kinase 1 and cyclin B1, and an increased p21 and p27 expression. Hoechst staining revealed chromatin condensation and nuclear fragmentation in the cells treated with cerdulatinib, and an increased fraction of apoptotic APO2.7-stained cells was detected by flow cytometry. This corresponded to the activation of caspase- $8,-9$ and -3 , and decreased levels of
\end{abstract}

Correspondence to: Professor Naoki Mori, Department of Microbiology and Oncology, Graduate School of Medicine, University of The Ryukyus, 207 Uehara, Nishihara, Okinawa 903-0215, Japan E-mail: naokimori50@gmail.com

Key words: adult T-cell leukemia/lymphoma, human T-cell leukemia virus type 1, cerdulatinib, spleen tyrosine kinase, Janus kinase, signal transducer and activator of transcription, nuclear factor- $\kappa \mathrm{B}$, activator protein-1, AKT, extracellular signal-regulated kinase the anti-apoptotic factors, Bcl-xL, survivin, $\mathrm{X}$-linked inhibitor of apoptosis (XIAP) and c-FLIP. The cerdulatinib-induced decrease in cell viability was partly reversed by the caspase inhibitor, z-VAD-FMK. These anti-ATLL effects were associated with the suppression of SYK and JAK/STAT signaling, along with that of the downstream factors, AKT, ERK, activator protein-1 and nuclear factor- $\kappa \mathrm{B}$. Finally, oral dosing with cerdulatinib lowered the tumor burden in a murine model of ATLL. Thus, our findings indicate that the simultaneous inhibition of therapeutically relevant targets, such as SYK and JAK is a more effective approach than single-agent therapy for the treatment of ATLL.

\section{Introduction}

Adult T-cell leukemia/lymphoma (ATLL) constitutes an aggressive T-cell malignancy characterized by the clonal expansion of $\mathrm{CD}^{+}{ }^{+} \mathrm{T}$-cells that develops in $2-7 \%$ of individuals infected with the retrovirus human T-cell leukemia virus type 1 (HTLV-1) (1). ATLL is associated with a poor prognosis, and despite advances in chemotherapy, the median survival time (8.3 and 10.6 months for acute and lymphoma types, respectively) has not improved since HTLV-1 was first described (2). Therefore, there is an urgent need for more effective therapies based on a detailed understanding of the molecular pathogenesis of this disease.

T-cells transformed by HTLV-1 are initially dependent on interleukin (IL)-2 for survival; however, these cells can subsequently transition into an IL-2-independent state (3). The IL-2 receptor (IL-2R) is composed of three subunits: IL-2R $\alpha$ and $-2 R \beta$, and the common $\gamma$ chain, with the cytoplasmic regions of IL-2R $\beta$ and the common $\gamma$ chain being critical for IL-2 signal transduction. Although they lack intrinsic protein tyrosine kinase (PTK) domains, these subunits recruit various non-receptor-type PTKs, such as Janus kinases (JAKs) and spleen tyrosine kinase (SYK) (4). In turn, JAK1 and JAK3 activate signal transducer and activator of transcription (STAT)1, STAT3 and STAT5 (5). Notably, the transition to IL-2 independence is associated with the activation of the JAK/STAT signaling pathway $(6,7)$, which is constitutively activated in HTLV-1-transformed T-cell lines, as well as primary ATLL cells (6-8). Accordingly, JAK inhibitors have been shown to 
inhibit the proliferation of ATLL cells $(9,10)$. SYK is also overexpressed in HTLV-1-transformed T-cell lines (11). Thus, therapeutic strategies that target JAK/STAT signaling and SYK may potentially benefit patients with ATLL.

Cerdulatinib is an orally available, ATP-competitive smallmolecule inhibitor of SYK and JAK1/3 kinases (12) that has been shown to exhibit antitumor activity in B-cell malignancies, including diffuse large B-cell lymphoma, Burkitt lymphoma and chronic lymphocytic leukemia (CLL) (12-15). A phase I/II dose escalation study in CLL, small lymphocytic lymphoma and non-Hodgkin lymphoma is currently underway (16). In the present study, we investigated the effects of dual SYK/JAK inhibition on the viability of HTLV-1-transformed and ATLLderived T-cell lines in order to evaluate the therapeutic potential of this approach.

\section{Materials and methods}

Reagents. Cerdulatinib (cat. no. S7634; Selleck Chemicals, Houston, TX, USA), JAK inhibitor 1 (cat. no. 420099; Merck Millipore, Burlington, MA, USA), PRT060318 (cat. no. SYN-1204; SYNkinase, Parkville, Australia) and z-VAD-FMK (cat. no. G7232; Promega Corp., Madison, WI, USA) were dissolved in dimethyl sulfoxide (cat. no. 13407-45; Nacalai Tesque, Inc., Kyoto, Japan). Antibodies against SYK (cat. no. 2712), phospho-SYK (Tyr525/526; cat. no. 2710), STAT3 (cat. no. 9132), phospho-STAT3 (Tyr705; cat. no. 9145), STAT5 (cat. no. 9363), phospho-STAT5 (Tyr694; cat. no. 4322), ERK (cat. no. 4695), phospho-ERK (Thr202/Tyr204; cat. no. 4370), phospho-p38 (Thr180/Tyr182; cat. no. 9211), Bcl-xL (cat. no. 2762), Bax (cat. no. 2772), Bak (cat. no. 3814), survivin (cat. no. 2808), AKT (cat. no. 9272), phospho-AKT (Thr308; cat. no. 13038 and Ser473; cat. no. 4060), phospho-IкB $\alpha$ (Ser32/36; cat. no. 9246), IкB kinase (IKK) $\alpha$ (cat. no. 2682), IKK $\beta$ (cat. no. 2684), phospho-IKK $\alpha / \beta$ (Ser176/180 and Ser177/181; cat. no. 2694), cleaved caspase-8 (cat. no. 9496), cleaved caspase-9 (cat. no. 9501), cleaved caspase-3 (cat. no. 9664), cleaved poly(ADP-ribose) polymerase (PARP; cat. no. 9541) and c-FLIP (cat. no. 3210) were from Cell Signaling Technology, Inc. (Beverly, MA, USA). Antibodies against cyclin B1 (cat. no. MS-868), cyclin-dependent kinase (CDK)1 (cat. no. MS-275), p53 (cat. no. MS-738) and actin (cat. no. MS-1295) were from Neomarkers, Inc. (Fremont, CA, USA). Antibodies against p21 (cat. no. MS-891), p27 (cat. no. MS-256) and X-linked inhibitor of apoptosis (XIAP; cat. no. M044-3) were from Medical \& Biological Laboratories, Co. (Aichi, Japan). Antibodies against JAK1 (cat. no. sc-277), phospho-JAK1 (Tyr1022/1023; cat. no. sc-16773-R), ІкB $\alpha$ (cat. no. sc-371), Mcl-1 (cat. no. sc-819) and c-IAP2 (cat. no. sc-7944) were from Santa Cruz Biotechnology, Inc. (Dallas, TX, USA).

Cell culture. HTLV-1-infected T-cell lines include HTLV-1transformed T-cell lines and ATLL-derived T-cell lines. HTLV-1-transformed MT-2 and HUT-102, ATLL-derived TL-OmI, and uninfected control Jurkat and CCRF-CEM T-cell lines were grown in RPMI-1640 medium (cat. no. 30264-56, Nacalai Tesque, Inc.) supplemented with $10 \%$ fetal bovine serum (Biological Industries, Kibbutz Beit Haemek, Israel) and $1 \%$ penicillin/streptomycin (cat. no. 09367-34; Nacalai
Tesque, Inc.) at $37^{\circ} \mathrm{C}$ and $5 \% \mathrm{CO}_{2}$ in a humidified incubator. The HUT-102 and Jurkat cells were obtained from Fujisaki Cell Center, Hayashibara Laboratories, Inc. (Okayama, Japan). The MT-2 cell line was kindly provided by Dr Naoki Yamamoto (Tokyo Medical and Dental University, Tokyo, Japan). The TL-OmI and CCRF-CEM cells were provided by Dr Masahiro Fujii (Niigata University, Niigata, Japan). Frozen human peripheral blood mononuclear cells (PBMCs) (cat. no. HC-0001) were obtained from Lifeline Cell Technology (Frederick, MD, USA).

Cell growth and cytotoxicity assay. The cells were seeded in triplicate in 96-well flat microtiter plates and treated with cerdulatinib $(0.16-10 \mu \mathrm{M})$, PRT060318 $(0.63-10 \mu \mathrm{M})$ and JAK inhibitor $1(0.63-10 \mu \mathrm{M})$ for 24-72 h. Cell growth and cytotoxicity were determined with the water-soluble tetrazolium (WST)-8 uptake assay using a kit (Nacalai Tesque, Inc.) according to the manufacturer's instructions. Briefly, $10 \mu \mathrm{l}$ of WST-8 reagent (cat. no. 07553-44; Nacalai Tesque, Inc.) was added to each well. After $4 \mathrm{~h}, \mathrm{WST}-8$ reduction was measured at $450 \mathrm{~nm}$ using a Wallac 1420 Multilabel Counter (PerkinElmer, Inc., Waltham, MA, USA). The absorbance values were normalized to those of the untreated control samples. The $50 \%$ inhibitory concentration $\left(\mathrm{IC}_{50}\right)$ for WST-8 activity was calculated by fitting data points with a logarithmic curve using CalcuSym software (version 2.0; Biosoft, Cambridge, UK).

Combination synergy calculation. Serial dilutions of JAK inhibitor 1 or PRT060318 alone, or in combination were tested. The combination index (CI) was calculated using CalcuSym software, with $\mathrm{CI}<1, \mathrm{CI}=1$ and $\mathrm{CI}>1$ representing synergistic, additive or antagonistic interactions of the two agents, respectively.

Cell cycle analysis. Cell cycle distribution was determined by staining with propidium iodide to label nuclear DNA using the CycleTEST Plus DNA Reagent kit (cat. no. 340242; Becton-Dickinson Immunocytometry Systems, San Jose, CA, USA) according to the supplier's instructions. The cells were sorted on an Epics XL flow cytometer (Beckman Coulter, Inc., Brea, CA, USA) and MultiCycle software (version 3.0; Phoenix Flow Systems, San Diego, CA, USA) was used to determine the fraction of cells at each phase of the cell cycle.

Analysis of cell apoptosis. The cells were treated with cerdulatinib $(2.5-10 \mu \mathrm{M})$ for up to $72 \mathrm{~h}$ and then permeabilized by incubation on ice for $20 \mathrm{~min}$ with $100 \mu \mathrm{g} / \mathrm{ml}$ of digitonin, followed by treatment with phycoerythrin-conjugated antiAPO2.7 antibody (1:10) (cat. no. IM2088; Beckman Coulter, Inc., Marseille, France) for $15 \mathrm{~min}$ at room temperature. The apoptotic fraction was separated by flow cytometry. To evaluate changes in nuclear morphology, the cells were stained with $10 \mu \mathrm{g} / \mathrm{ml}$ Hoechst 33342 (cat. no. 346-07951; Dojindo Molecular Technologies, Inc., Kumamoto, Japan) and observed under a DMI6000 microscope (Leica Microsystems, Wetzlar, Germany) (17).

Measurementofcaspase activity. Caspaseactivity was measured using Colorimetric Caspase Assay kits (cat. nos. 4800, 4805 and 4810, Medical \& Biological Laboratories, Co.). Briefly, cell 
extracts were recovered using the cell lysis buffer supplied with the kit, and caspase- $8,-9$ and -3 activity was assessed using the specific colorimetric probe. The kits are based on detection of the chromophore $\mathrm{Q}$-nitroanilide after cleavage from caspasespecific labeled substrates. Colorimetric measurements were made with a Wallac 1420 Multilabel Counter.

Western blot analysis. The cells were lysed in lysis buffer containing 62.5 mM Tris- $\mathrm{HCl}$ (pH 6.8) (cat.no. 35434-21; Nacalai Tesque,Inc.), 2\% sodium dodecyl sulfate (SDS) (cat. no. 31607-65; Nacalai Tesque, Inc.), $10 \%$ glycerol (cat. no. 17045-65; Nacalai Tesque, Inc.), 6\% 2-mercaptoethanol (cat. no. 21438-82; Nacalai Tesque, Inc.) and $0.01 \%$ bromophenol blue (cat. no. 021-02911; Wako Pure Chemical Industries, Osaka, Japan). The protein concentration was determined using the DC Protein Assay kit (cat. no. 5000116JA; Bio-Rad Laboratories, Inc., Hercules, CA, USA). Cell lysates $(20 \mu \mathrm{g})$ were separated by $8-15 \%$ SDS-polyacrylamide gel electrophoresis and transferred onto a polyvinylidene difluoride membrane (cat. no. IPVH00010EMD; Merck KGaA, Darmstadt, Germany) that was probed with primary antibodies $(1: 1,000)$. The membranes were blocked with 4\% non-fat dry milk (cat. no. 9999; Cell Signaling Technology, Inc.) for $1 \mathrm{~h}$ at room temperature. Following incubation with horseradish peroxidase-conjugated secondary anti-mouse (1:1,000) (cat. no. 7076; Cell Signaling Technology, Inc.) or antirabbit $(1: 1,000)$ (cat. no. 7074; Cell Signaling Technology, Inc.) IgG, immunoreactivity was visualized using enhanced chemiluminescence reagent (cat. no. RPN2232; Amersham Biosciences Corp., Piscataway, NJ, USA).

Electrophoretic mobility shift assay (EMSA). To assess STAT3, STAT5, nuclear factor- $\kappa \mathrm{B}(\mathrm{NF}-\kappa \mathrm{B})$ and activator protein-1 (AP-1) activation, we prepared nuclear extracts from the cerdulatinib-treated cells and performed EMSA, as previously described (18). Nuclear extracts with $5 \mu \mathrm{g}$ of protein were incubated with ${ }^{32} \mathrm{P}$-labeled probes. The top strand sequences of the oligonucleotide probes were as follows: 5'-GATCGACATTTCCCGTAAATCG-3' (STAT3 consensus binding motif derived from the c-fos gene); 5'-GATCAGAT TTCTAGGAATTCAAATC-3' (STAT5 consensus binding motif derived from the CSN2 gene); 5'-GATCCGGCAG GGGAATCTCCCTCTC-3' (typical NF- $\kappa$ B element of the $I L-2 R A$ gene); and 5'-GATCGTGATGACTCAGGTT-3' (consensus AP-1 element of the $I L-8$ gene). The oligonucleotide 5'-GATCTGTCGAATGCAAATCACTAGAA-3' containing the consensus sequence of the octamer (Oct) binding motif was used to evaluate specific binding of the transcription factor Oct-1, which regulates the transcription of a number of housekeeping genes. STAT3, STAT5, NF- $\kappa$ B, AP-1 and Oct-1 binding sites are underlined in the above sequences. It has been reported that NF- $\mathrm{B}$ (p50, RelA and RelB), AP-1 (JunB and JunD), STAT3 and STAT5 specifically bind to the above oligonucleotides in HTLV-1-infected T-cell lines and ATLL cells $(8,19)$.

Xenograft tumor model. C.B-17/Icr-severe combined immune deficient (SCID) mice are a useful model for investigating the proliferative and tumorigenic potential of HTLV-1-infected T-cell lines. HUT-102 cells (1x107/0.2 ml RPMI-1640 medium) were subcutaneously transplanted into the postauricular region of 5-week-old female SCID mice (Kyudo, Co., Tosu, Japan) on day 0. The mouse body weight was 16.6-18.3 $\mathrm{g}$ upon purchase. Animal cages were maintained at a temperature of $24^{\circ} \mathrm{C}$ and a humidity of $60 \%$. The mice were fed a standard rodent diet (CE-2; CLEA Japan, Inc., Tokyo, Japan) and water ad libitum. The mice were divided into 2 groups $(n=5$, each). Cerdulatinib was solubilized in $0.5 \%$ methylcellulose (cat. no. 133-17815; Wako Pure Chemical Industries) and administered at a dose of $35 \mathrm{mg} / \mathrm{kg}$ by oral gavage 5 times a week. The treatment was continued for up to 28 days starting from the day after cell inoculation. The control group received the vehicle $(0.3 \mathrm{ml}$ of $0.5 \%$ methylcellulose) only. The tumor diameter was measured weekly with a shifting caliper and tumor volume was calculated using the ellipsoid volume formula ( $\pi / 6 \times$ length $\mathrm{x}$ width $\mathrm{x}$ height) (20). Body weight was also measured weekly. The mice were sacrificed on day 28 . Tumors were collected and their weight was measured. Experimental procedures and animal care were in compliance with the Guidelines for Animal Experimentation of University of The Ryukyus (Nishihara, Japan) and were approved by the Animal Care and Use Committee of University of The Ryukyus (reference no. A2016088).

Morphological analysis of tumor tissue and terminal deoxynucleotidyl transferase deoxyuridine triphosphate nick-end labeling (TUNEL) assay. Tumor specimens were collected from mice, fixed in formalin (Wako Pure Chemical Industries) solution, dehydrated through a graded ethanol series (Japan Alcohol Selling Co., Tokyo, Japan) and embedded in paraffin (cat. no. 09620; Sakura Finetek Japan Co., Tokyo, Japan). The specimens were cut into sections that were stained with hematoxylin and eosin (H\&E; cat. nos. 234-12 and 1159350025; Merck $\mathrm{KGaA}$ ) for histological examination. DNA fragmentation was analyzed with the TUNEL assay using a commercial kit (cat. no. 11684817910; Roche Applied Science, Penzberg, Germany) according to the manufacturer's instructions. Cells were examined under a light microscope (Axioskop 2 Plus) with an Achroplan 40x/0.65 lens (both from Zeiss, Hallbergmoos, Germany). Images were acquired with an AxioCam 503 color camera and AxioVision LE64 software (Zeiss GmbH, Jena, Germany).

Statistical analysis. Results are expressed as the means \pm standard deviation (SD). The Student's t-test or ANOVA with the Tukey-Kramer test were used to compare the means of 2 groups or $>2$ groups, respectively. Differences were considered statistically significant at $\mathrm{P}<0.05$.

\section{Results}

HTLV-1-infected T-cell lines are sensitive to dual SYK/JAK inhibition with cerdulatinib. Using the WST-8 assay, we first examined the cytotoxicity of various concentrations of cerdulatinib in HTLV-1-infected T-cell lines up to $72 \mathrm{~h}$ after treatment (Fig. 1A). Cerdulatinib decreased WST-8 activity (and thus, cell viability) in a dose- and time-dependent manner in all HTLV-1-infected T-cell lines. Notably, the uninfected Jurkat and CCRF-CEM T-cell lines were resistant to the cytotoxic effects of cerdulatinib. The effects of cerdulatinib on PBMCs from a healthy donor were less pronounced (Fig. 1B). 
A
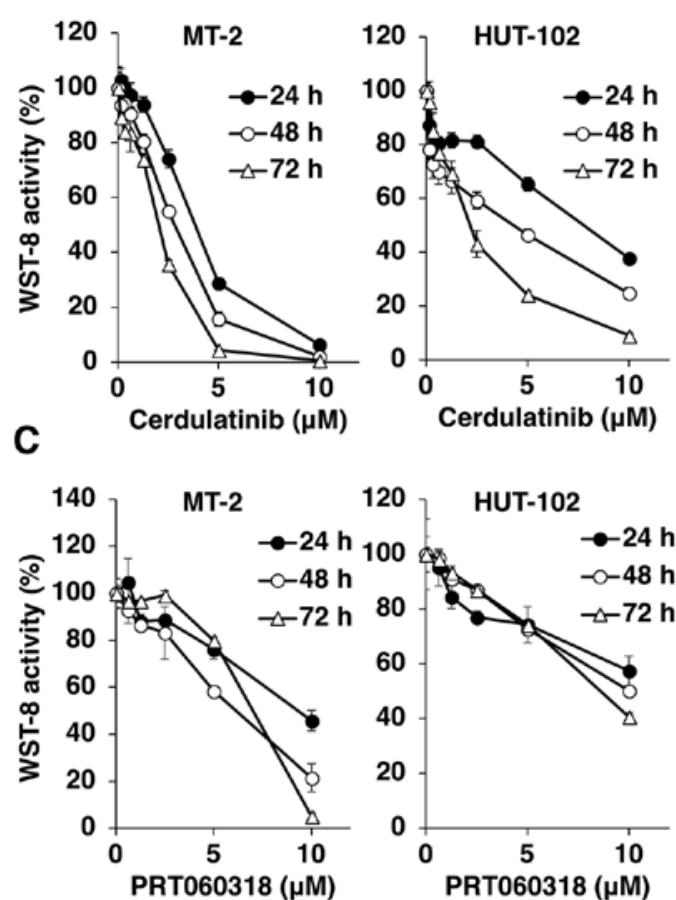

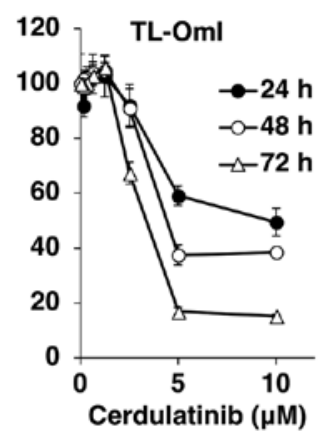

B
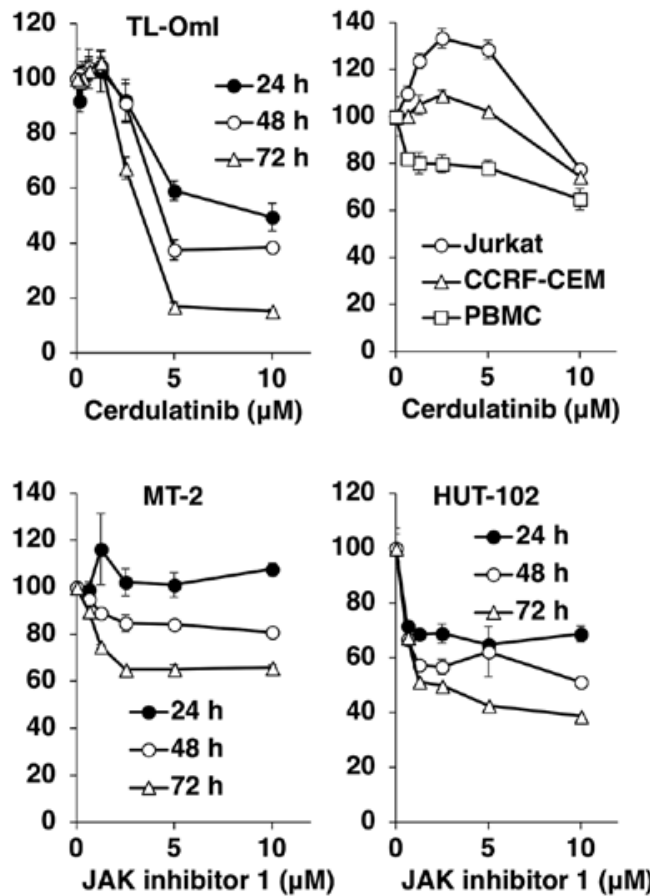

Figure 1. HTLV-1-infected T-cell lines are sensitive to dual SYK/JAK inhibition by cerdulatinib. (A) Cells were treated with various concentrations of cerdulatinib for 24-72 $\mathrm{h}$ and cell viability was evaluated by WST-8 assay. (B) Two uninfected T-cell lines were treated with increasing concentrations of cerdulatinib. PBMCs from a healthy donor were included as a normal cell control. After $24 \mathrm{~h}$, cell viability was evaluated by WST-8 assay. (C) MT- 2 and HUT-102 cells were incubated with the indicated concentrations of PRT060318 and JAK inhibitor 1, and viability was assessed after 24-72 h. Data are presented as the means \pm SD of triplicate cultures.

The $\mathrm{IC}_{50}$ values at $24 \mathrm{~h}$ were $3.5 \mu \mathrm{M}$ for the MT-2, $7.3 \mu \mathrm{M}$ for the HUT-102 and $8.4 \mu \mathrm{M}$ for the TL-OmI cells, as compared to $62.9 \mu \mathrm{M}$ for the normal PBMCs. In the Jurkat and CCRF-CEM cells, the $\mathrm{IC}_{50}$ value was not attained with the administered doses. We compared the effects of cerdulatinib with those of two reference compounds, the SYK-selective inhibitor, PRT060318, and the pan-JAK inhibitor, JAK inhibitor 1, on the MT-2 and HUT-102 cells, and found that cerdulatinib decreased cell viability more potently than either single agent (Fig. 1, compare parts A and C). As PRT060318 or JAK inhibitor 1 decreased the viability of HTLV-1-infected T-cell lines, we further tested the combination of the two agents to determine whether they acted synergistically to suppress the viability of HTLV-1-infected T-cells. The CI was used to determine whether the effect of the combined treatment was synergistic, additive or antagonistic. Instead of a simple additive effect, the combination of PRT060318 and JAK inhibitor 1 exerted a synergistic cytotoxic effect on HUT-102 cells, with the optimal CI of 0.609 (Fig. 2).

Cerdulatinib inhibits cell cycle progression. The negative effect of cerdulatinib on cell viability (as determined using the WST-8 assay) may result from cell growth inhibition, death or both. To clarify the mechanisms underlying the inhibition of cell growth by cerdulatinib, we evaluated cell cycle distribution in the MT-2, HUT-102 and TL-OmI cells treated with 2.5 and $5 \mu \mathrm{M}$ cerdulatinib for $24 \mathrm{~h}$. Treatment with cerdulatinib led to a significant accumulation of cells in the $\mathrm{G}_{2} / \mathrm{M}$-phase, with concurrent decreases in the $\mathrm{G}_{1}$ - and/or S-phase fractions (Fig. 3). These results indicated that cerdulatinib inhibits cell
A

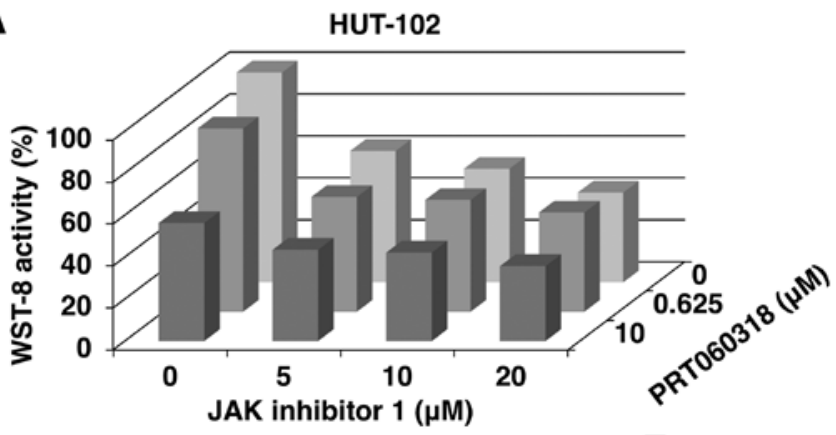

B

\begin{tabular}{|c|c|c|c|}
\hline \multicolumn{3}{|c|}{ Combination index } & \multirow{3}{*}{$\begin{array}{l}0.62 \\
10\end{array}$} \\
\hline 0.609 & 1.049 & 1.359 & \\
\hline 0.683 & 0.859 & 0.834 & \\
\hline 5 & 10 & 20 & \\
\hline
\end{tabular}

Figure 2. JAK and SYK inhibition synergistically inhibits the viability of HTLV-1-infected T-cells. (A) HUT-102 cells were treated with various concentrations of JAK inhibitor 1, PRT060318 or their combination at indicated concentrations. After $24 \mathrm{~h}$, cell viability was evaluated with the WST-8 assay. (B) CI was calculated from the data shown in (A) using CalcuSym software.

proliferation by targeting the $\mathrm{G}_{2} / \mathrm{M}$ checkpoint and blocking cell cycle progression.

Cerdulatinib induces apoptosis. We then examined the effect of cerdulatinib on cell death. A morphological analysis using Hoechst staining revealed chromatin condensation and nuclear fragmentation in the cells cultured with cerdulatinib (Fig. 4A). 
A

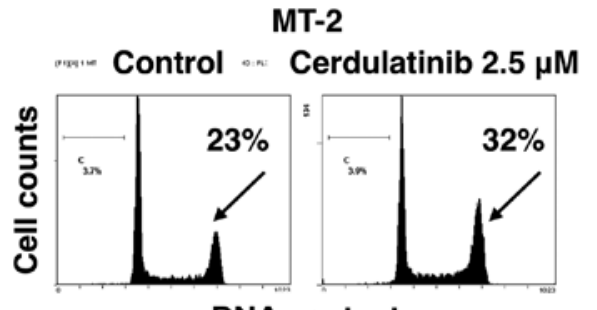

HUT-102

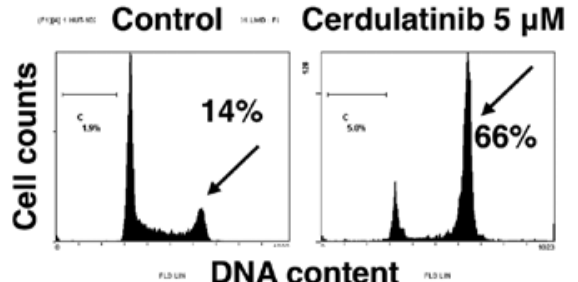

TL-Oml

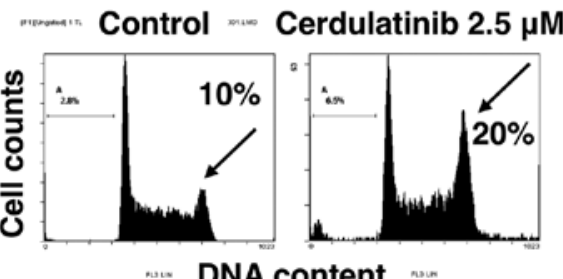

B

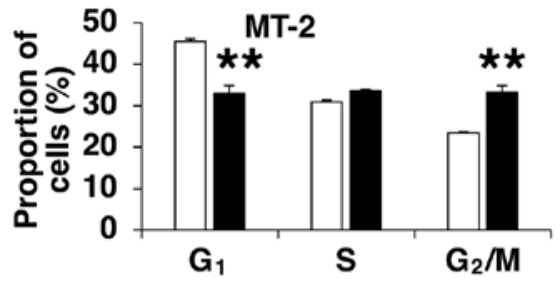

$\square$ Control $\backsim$ Cerdulatinib $2.5 \mu \mathrm{M}$

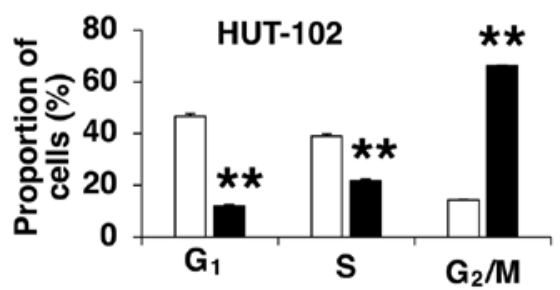

$\square$ Control $\square$ Cerdulatinib $5 \mu \mathrm{M}$

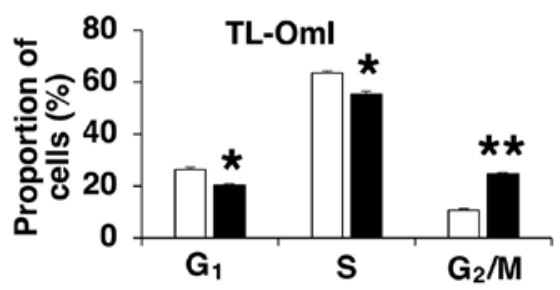

$\square$ Control $\backsim$ Cerdulatinib $2.5 \mu \mathrm{M}$

Figure 3. Effect of cerdulatinib on cell cycle distribution. MT-2, HUT-102 and TL-OmI cells were treated for 24 h with the indicated concentrations of cerdulatinib prior to staining with propidium iodide and analysis by flow cytometry. (A) Representative histograms are shown and the percentages of cells in $\mathrm{G}_{2} / \mathrm{M}$-phase are indicated. (B) Quantification of the percentage of cells in each phase of the cell cycle. Values are presented as the means \pm SD of triplicate cultures. ${ }^{*} \mathrm{P}<0.001$ and ${ }^{* *} \mathrm{P}<0.0005$, vs. vehicle-treated control.

A

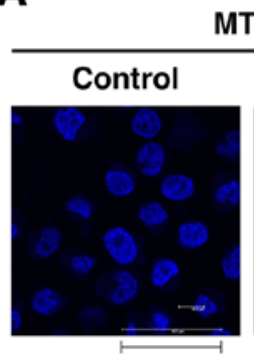

B
MT-2

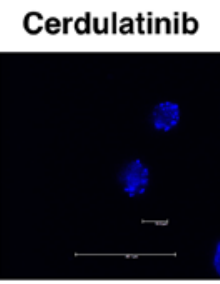

$40.1 \mu \mathrm{m}$

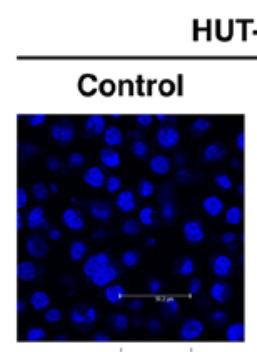

$50.2 \mu \mathrm{m}$
HUT-102

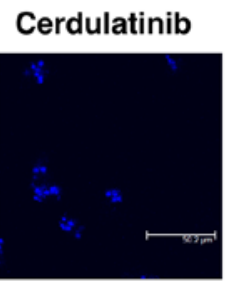

$50.2 \mu \mathrm{m}$

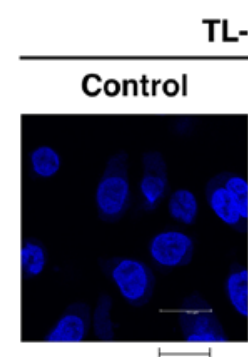

$15.0 \mu \mathrm{m}$
TL-Oml

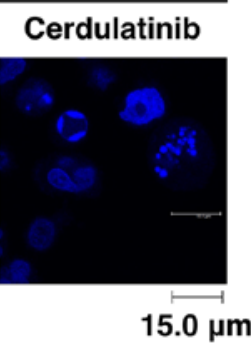

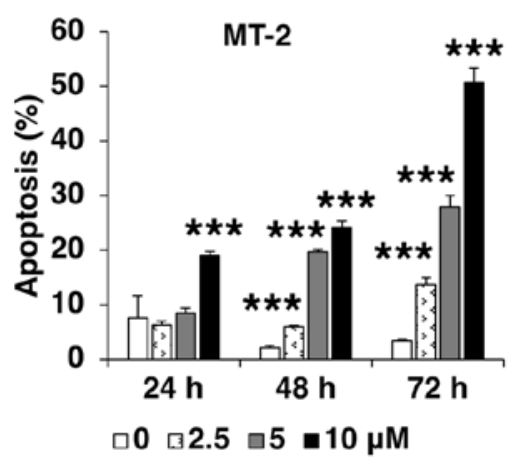
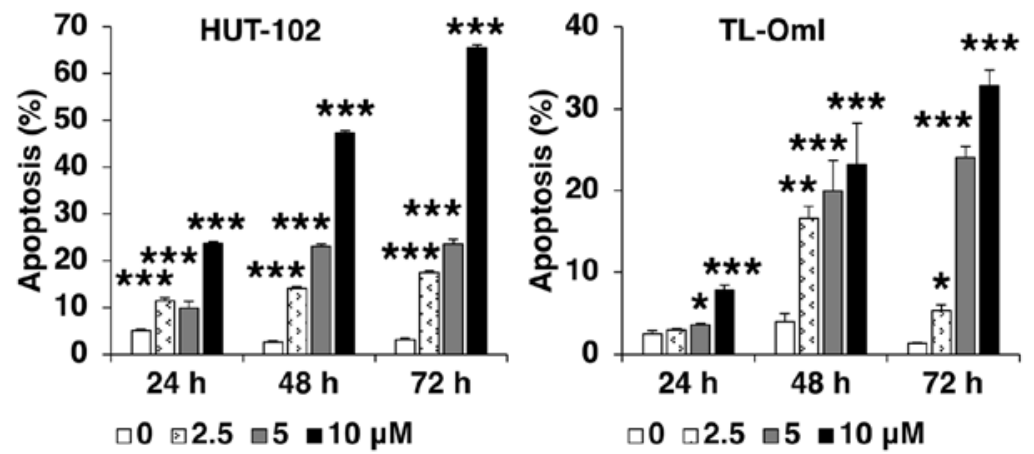

Figure 4. Cerdulatinib induces the apoptosis of HTLV-1-infected T-cell lines. (A) Photomicrographs of representative fields of cells stained with Hoechst 33342 dye showing chromatin condensation and nuclear fragmentation after $48 \mathrm{~h}$ of treatment with $10 \mu \mathrm{M}$ cerdulatinib. (B) Quantitative analysis of apoptotic cells. Cells were treated for 24-72 $\mathrm{h}$ with the indicated concentrations of cerdulatinib prior to staining with APO2.7 and analysis by flow cytometry. Data are presented as the means \pm SD of triplicate cultures. ${ }^{*} \mathrm{P}<0.05,{ }^{* *} \mathrm{P}<0.01$ and ${ }^{* * *} \mathrm{P}<0.005$, vs. vehicle-treated control. 

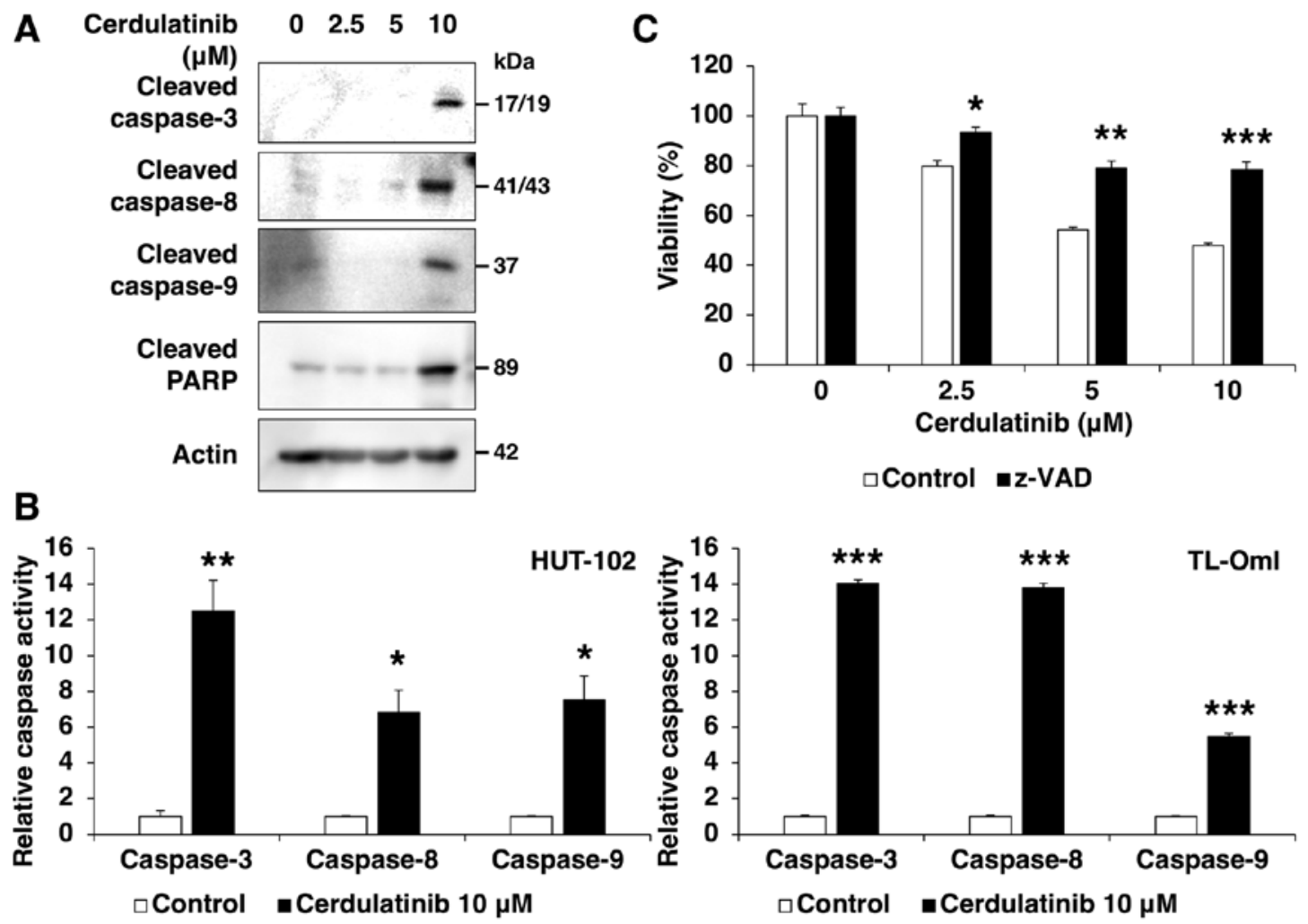

Figure 5. Effect of cerdulatinib on caspases. (A) Cleaved caspase-8, -9 and -3, and PARP protein levels in cells treated with cerdulatinib were determined by western blot analysis. HUT-102 cells were treated with the indicated concentrations of cerdulatinib for $48 \mathrm{~h}$, and lysates were used for western blot analysis. Actin served as a loading control. (B) Cells were treated with $10 \mu \mathrm{M}$ cerdulatinib for $72 \mathrm{~h}$; lysates were incubated with caspase-specific labeled substrate, and colorimetric products were measured. ${ }^{*} \mathrm{P}<0.005,{ }^{* *} \mathrm{P}<0.0005$ and ${ }^{* * * *} \mathrm{P}<0.0001$, vs. vehicle-treated control. (C) TL-OmI cells were pre-treated with the pan-caspase inhibitor z-VAD-FMK followed by treatment with the indicated concentrations of cerdulatinib for $48 \mathrm{~h}$. Cell viability was assessed by WST-8 assay. Data are presented as the means \pm SD of triplicate cultures. ${ }^{*} \mathrm{P}<0.005,{ }^{* *} \mathrm{P}<0.0005$ and ${ }^{* * *} \mathrm{P}<0.0001$, for cerdulatinib with vs. without $\mathrm{z}$-VAD-FMK pre-treatment.

APO2.7 immunolabeling indicated that apoptosis was induced by cerdulatinib in a concentration- and time-dependent manner (Fig. 4B). To investigate the role of caspases in the response to cerdulatinib, cell lysates were examined by western blot analysis using specific antibodies. The induction of apoptosis of the HUT-102 cells at increasing concentrations of cerdulatinib was accompanied by the cleavage of caspase- $8,-9$ and -3 , and its substrate PARP (Fig. 5A). We also examined the enzymatic activity of caspase-8, -9 and -3 , as this is not always associated with pro-caspase processing, and found the significant activation of all three proteins in the HUT-102 and TL-OmI cells following $72 \mathrm{~h}$ of treatment with $10 \mu \mathrm{M}$ cerdulatinib (Fig. 5B). These results suggested that cerdulatinib induces apoptosis via the activation of extrinsic and intrinsic pathways. Pre-treatment with the pan-caspase inhibitor, z-VAD-FMK, partly abrogated cerdulatinib-induced cytotoxicity (Fig. 5C), suggesting that the latter occurs through caspase-dependent apoptosis.

Cerdulatinib blocks SYK, JAK/STAT, AKT, ERK, NF- $\kappa B$ and $A P-1$ signaling. To determine whether cerdulatinib inhibits the SYK and JAK/STAT signaling pathways, we evaluated the phosphorylation status of SYK and JAK-STAT in the HUT-102 cells treated with cerdulatinib by western blot analysis, and found that SYK, JAK1, STAT3 and STAT5 phosphorylation was decreased (Fig. 6). SYK is associated with various immunoreceptors (21) and is thought to act upstream of phosphoinositide 3-kinase (PI3K)-AKT-mediated
IKK-I $\kappa \mathrm{B} \alpha$ activation (21-23). In addition, JAK inhibition has been reported to suppress IL-2-mediated T-cell growth and STAT, AP-1 and ERK activation by IL-2 (24). In this study, we found that cerdulatinib blocked the phosphorylation of AKT, IKK $\alpha / \beta$, IKB $\alpha$ and ERK, but not that of p38 (Fig. 6), and nuclear extracts obtained from the cerdulatinib-treated cells exhibited a reduced DNA binding of STAT3, STAT5, NF- $\kappa$ B and AP-1, but not Oct-1 (Fig. 7A). Thus, SYK and JAK/STAT, as well as downstream signaling pathways were effectively inhibited by cerdulatinib.

Cerdulatinib modulates key proteins related to cell cycle progression and apoptosis. To further clarify the molecular mechanisms underlying the growth inhibitory and cytotoxic effects of cerdulatinib on HTLV-1-infected T-cell lines, we evaluated the expression of proteins associated with cell cycle progression and apoptosis. The results of western blot analysis revealed that cerdulatinib treatment increased the expression of p21 and p27, and decreased that of CDK1, cyclin B1, Bcl-xL, survivin, XIAP and c-FLIP (Fig. 7B and C). However, it had no effect on the levels of Mcl-1, c-IAP2, and the pro-apoptotic proteins, Bak and Bax. As p21 and p27 expression is modulated by p53, we also examined p53 expression, but found no obvious changes in the level of this protein in the cerdulatinibtreated cells.

Pre-clinical evaluation of cerdulatinib in a mouse xenograft model of ATLL. We examined the in vivo efficacy of cerdulatinib 


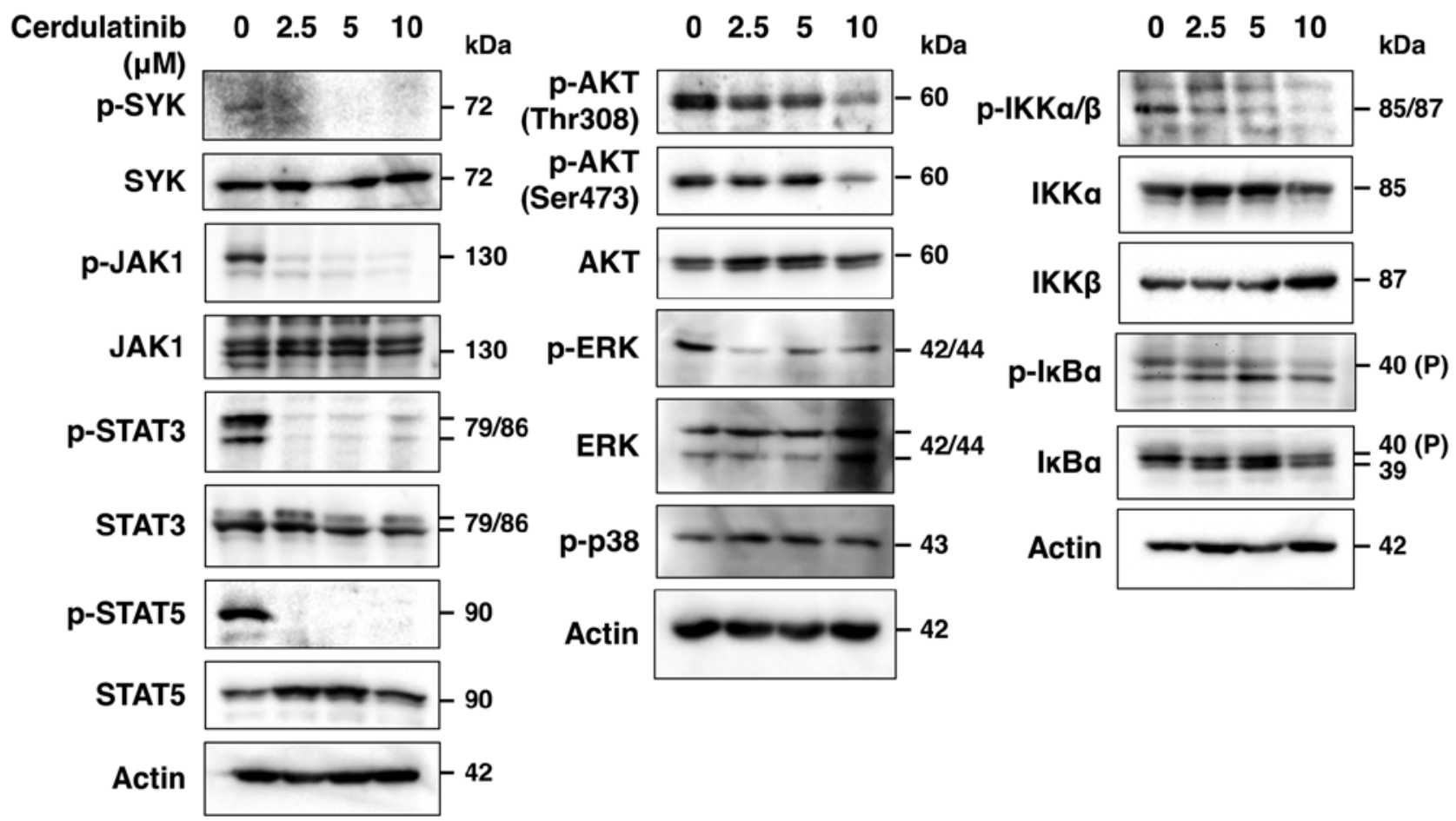

Figure 6. Effects of cerdulatinib on the expression of intracellular signaling pathway components in HUT-102 cells. Cells were treated with the indicated concentrations of cerdulatinib. After $48 \mathrm{~h}$, cell lysates were analyzed by western blot analysis. The positions of IкB $\alpha$ and the phosphorylated form of the protein $(\mathrm{P})$ are indicated. Actin served as a loading control.

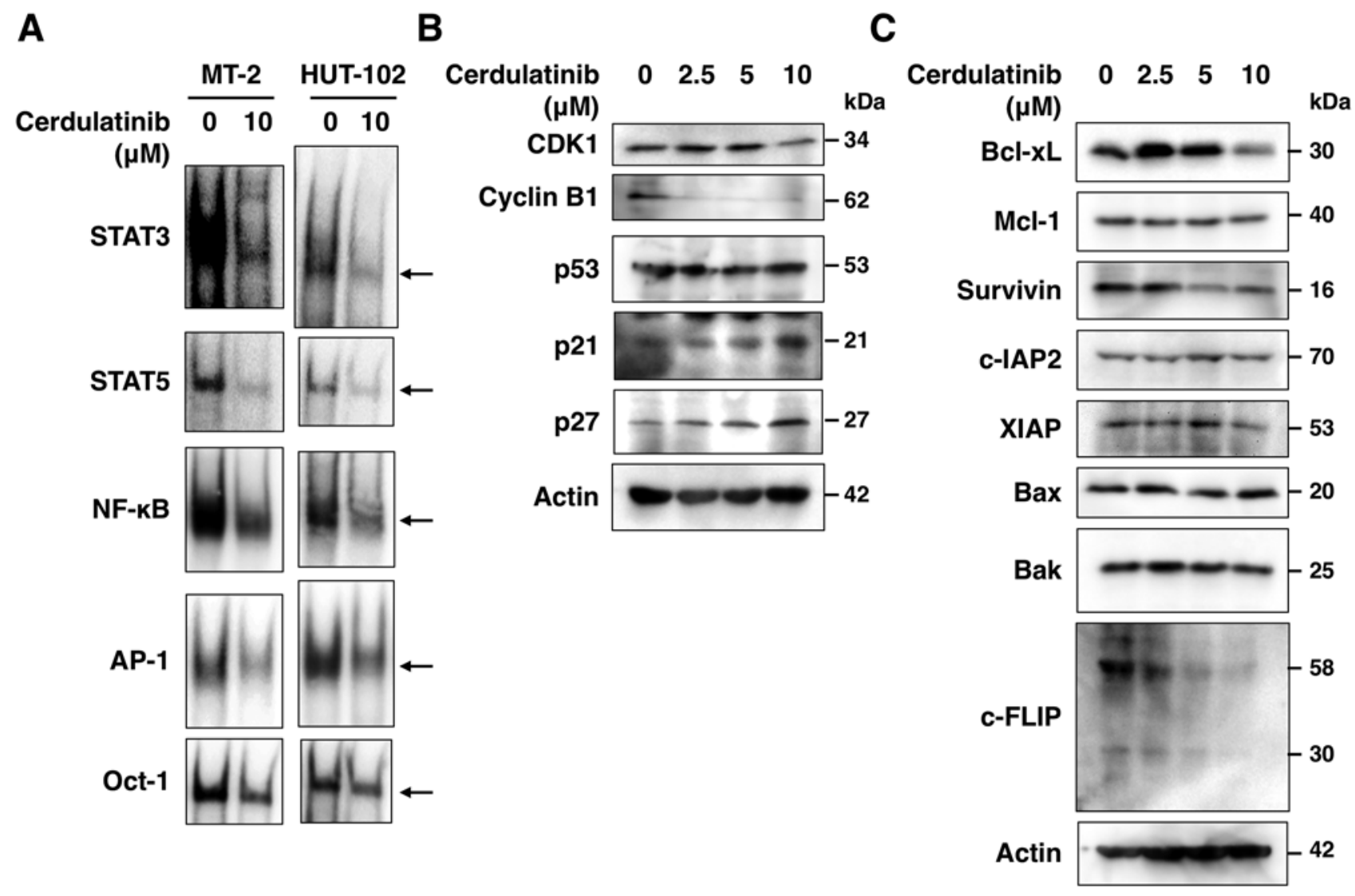

Figure 7. Effects of cerdulatinib on DNA binding of transcription factors and the expression of proteins related to cell cycle progression and apoptosis. (A) Cerdulatinib treatment inhibited the DNA binding of STAT3, STAT5, NF- $\mathrm{kB}$ and AP-1, as demonstrated by EMSA. MT-2 and HUT-102 cells were cultured in the absence or presence of $10 \mu \mathrm{M}$ cerdulatinib for $48 \mathrm{~h}$. Nuclear extracts were incubated with ${ }^{32} \mathrm{P}$-labeled oligonucleotide probes corresponding to the DNA binding sites of STAT3, STAT5, NF- $\mathrm{KB}$ and AP-1. Arrows indicate each migrated DNA complex. Effects of cerdulatinib on the expression of proteins related to cell cycle progression (B) and apoptosis (C) in HUT-102 cells. Cells were treated with the indicated concentrations of cerdulatinib. After $48 \mathrm{~h}$, cell lysates were examined by western blot analysis. Actin served as a loading control. 
A

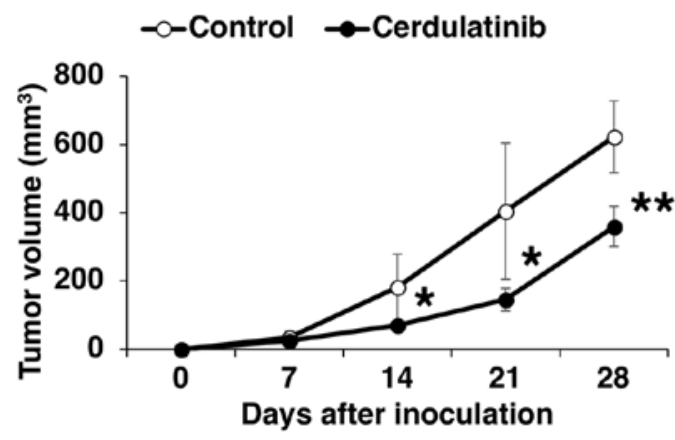

C

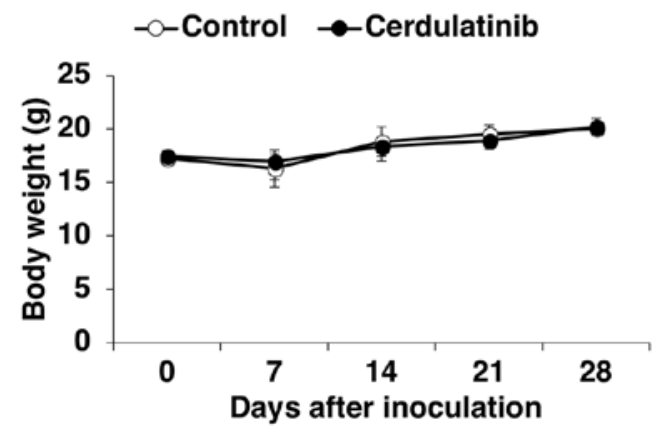

B

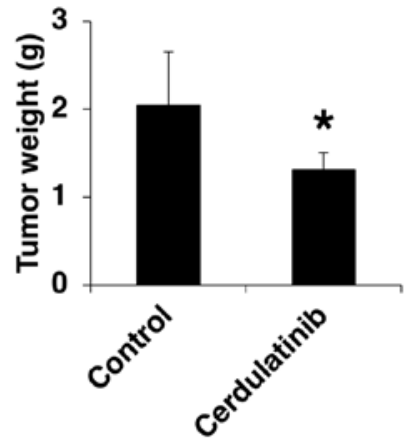

D

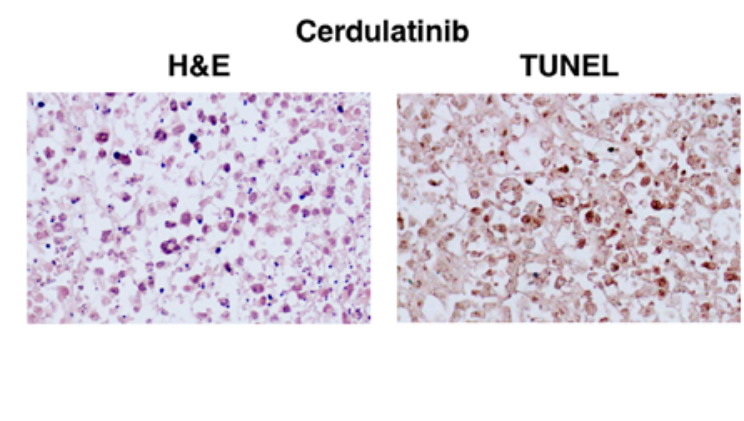

Figure 8. Anti-ATLL effect of cerdulatinib in a mouse xenograft model. HUT-102 cells were subcutaneously injected into the postauricular region of mice to establish xenograft models. The mice were then orally treated with either cerdulatinib (35 mg/kg) or vehicle (control) 5 times weekly for 28 days. (A and B) Tumor volume and weight in each group. ${ }^{*} \mathrm{P}<0.05$ and ${ }^{* *} \mathrm{P}<0.005$, vs. control group. (C) Changes in body weight over time in mice harboring HUT-102 cellderived tumors and treated with cerdulatinib. (D) H\&E staining and TUNEL staining in tumor tissue from mice treated with cerdulatinib. Magnification, $\mathrm{x} 800$

in a mouse xenograft model of ATLL. HUT-102 cells were subcutaneously transplanted into SCID mice, which were then orally administered the vehicle or $35 \mathrm{mg} / \mathrm{kg}$ cerdulatinib 5 times weekly. The volume and weight of the transplanted tumors were lower in the mice treated with cerdulatinib than in the control group (Fig. 8A and B). Cerdulatinib was well tolerated, as it did not cause significant weight loss or other clinical manifestations (Fig. 8C). Tumor tissue from the mice treated with cerdulatinib examined by H\&E staining (Fig. 8D, left panel) exhibited evidence of apoptosis, including cytoplasmic condensation, chromatin hyperchromatism and condensation, and nuclear fragmentation. Apoptosis in the tumor tissue from the cerdulatinib-treated mice was confirmed by TUNEL assay (Fig. 8D, right panel), which was consistent with our in vitro findings. These results demonstrated the effectiveness of cerdulatinib in controlling ATLL progression in vivo.

\section{Discussion}

In this study, we evaluated the therapeutic potential of cerdulatinib, a potent inhibitor of SYK and JAK1/3, in the treatment of ATLL. We demonstrated that this compound was effective in suppressing the growth of HTLV-1-infected T-cell lines in vitro and ATLL xenograft tumors in vivo. The HTLV-1infected T-cell lines were more sensitive to cerdulatinib than uninfected T-cell lines and normal PBMCs. Moreover, cerdulatinib exhibited greater cytotoxicity than either a SYK or JAK single inhibitor alone.
Cerdulatinib induced apoptosis in addition to causing cell cycle arrest in HTLV-1-infected T-cell lines. The inhibition of JAK can lead to the suppression of STAT, ERK and AP-1, thereby blocking the proliferation of IL-2-stimulated T-cells (24), whereas SYK is known to induce PI3K-AKT activation, which is critical for NF- $\kappa$ B signaling (21-23). In this study, we observed that cerdulatinib treatment suppressed both SYK and JAK-STAT signaling along with the activity of the downstream factors, AKT, ERK, AP-1 and NF- $\kappa$ B.

To investigate the mechanistic basis for the cell cycle arrest at the $\mathrm{G}_{2} / \mathrm{M}$ phase induced by cerdulatinib, we examined the CDK1 and cyclin B1 levels in cerdulatinib-treated cells, as the $\mathrm{CDK} 1 /$ cyclin B1 complex constitutes a major regulator of the $\mathrm{G}_{2} / \mathrm{M}$ transition. Cerdulatinib induced a marked decrease in the CDK1 and cyclin B1 levels. p21 and p27 are CDK inhibitors that negatively regulate cell cycle progression, with their suppression resulting in increased cell proliferation (25). p21 plays a well-documented role as a $\mathrm{G}_{1} / \mathrm{S}$ checkpoint protein, but is also known to modulate the $\mathrm{G}_{2} / \mathrm{M}$ checkpoint $(26,27)$ through the inhibition of the CDK1/cyclin B1 complex (28). p27 is an inhibitor of CDK1 (27). AKT, ERK, AP-1 and NF- $\mathrm{B}$ regulate cyclin B1 expression in cell proliferation $(29,30)$, and AKT and ERK have also been shown to suppress p21 and p27 (31-33). In this study, following treatment with cerdulatinib, the p21 and p27 protein levels were upregulated relative to those of the control group. Our results indicated that cerdulatinib suppressed HTLV-1-infected T-cell proliferation by the downregulation of CDK1 and cyclin B1, and the upregulation 
of the CDK inhibitors, p21 and p27, causing p53-independent cell cycle arrest at the $\mathrm{G}_{2} / \mathrm{M}$ phase by suppressing the AKT, ERK, AP-1 and NF- $\kappa$ B pathways.

Cerdulatinib blocked the expression of the anti-apoptotic factors, Bcl-xL, survivin, XIAP and c-FLIP, which prevent caspase activation; their inhibition therefore increases caspase-3, -8 and -9 levels $(34,35)$. STAT and NF- $\kappa$ B are translocated to the nucleus where they bind to specific DNA sequences in the promoters of target genes, including $B C L X L$, $B I R C 5$ and XIAP, thereby inducing their transcription $(36,37)$. c-FLIP expression is regulated by AKT and NF- $\kappa \mathrm{B}$ (35). AKT activates NF- $\kappa \mathrm{B}(22,31,37)$ but also inhibits caspase-9 activation along with that of ERK $(31,34,38)$. AKT activity is regulated by XIAP (39), whereas survivin is a downstream target of AKT signaling $(40,41)$. Thus, the increase in caspase activity in cerdulatinib-treated cells suggested that this compound promotes apoptosis by suppressing AKT, ERK, STAT and NF- $\kappa$ B pathways. Notably, the safety and clinical efficacy of cerdulatinib was recently demonstrated in patients with CLL, as well as in those with another type of B-cell non-Hodgkin lymphoma (42), further supporting its potential for application as a treatment for ATLL.

In conclusion, the findings of this study demonstrate that cerdulatinib exerts anti-ATLL effects in vitro and in vivo. Although the detailed mechanisms underlying the modulation of CDK1 by cerdulatinib require further investigation, our results indicate that cerdulatinib induces $\mathrm{G}_{2} / \mathrm{M}$ arrest and subsequent apoptosis by inhibiting the SYK and JAK/STAT signaling pathways and their downstream effectors, AKT, ERK, AP-1 and NF- $\kappa$ B. The preclinical findings presented herein provide a rationale for evaluating the efficacy of cerdulatinib in patients with ATLL.

\section{Acknowledgements}

The authors would like to thank the Fujisaki Cell Center, Hayashibara Biochemical Laboratories, Inc. (Okayama, Japan) for providing the HUT-102 and Jurkat cells, Dr Naoki Yamamoto (Tokyo Medical and Dental University, Tokyo, Japan) for providing MT-2 cells, Dr Masahiro Fujii (Niigata University, Niigata, Japan) for providing TL-OmI and CCRF-CEM cells, and Editage (www.editage.jp) for English language editing.

\section{Funding}

This study was supported in part by a JSPS KAKENHI grants (nos. 15K18414 and 17K07175).

\section{Availability of data and materials}

All data generated or analyzed during this study are included in this published article.

\section{Authors' contributions}

CI and NM were responsible for the study design, the drafting and editing of the manuscript, and data acquisition and analysis. MS was responsible for data acquisition and analysis. All authors have read and approved this manuscript.

\section{Ethics approval and consent to participate}

Experimental procedures and animal care were in compliance with the Guidelines for Animal Experimentation of University of The Ryukyus (Nishihara, Japan) and were approved by the Animal Care and Use Committee of University of The Ryukyus (reference no. A2016088).

\section{Patient consent for publication}

Not applicable.

\section{Competing interests}

The authors declare that they have no competing interests.

\section{References}

1. Iwanaga M, Watanabe T and Yamaguchi K: Adult T-cell leukemia: A review of epidemiological evidence. Front Microbiol 3: 322, 2012.

2. Katsuya $H$ and Ishitsuka $\mathrm{K}$ : Treatment advances and prognosis for patients with adult T-cell leukemia-lymphoma. J Clin Exp Hematop 57: 87-97, 2017.

3. Yssel H, de Waal Malefyt R, Duc Dodon MD, Blanchard D, Gazzolo L, de Vries JE and Spits H: Human T cell leukemia/ lymphoma virus type I infection of a CD4+ proliferative/ cytotoxic $\mathrm{T}$ cell clone progresses in at least two distinct phases based on changes in function and phenotype of the infected cells. J Immunol 142: 2279-2289, 1989.

4. Miyazaki T and Taniguchi T: Coupling of the IL2 receptor complex with non-receptor protein tyrosine kinases. Cancer Surv 27: 25-40, 1996

5. Rochman Y, Spolski R and Leonard WJ: New insights into the regulation of $\mathrm{T}$ cells by gamma(c) family cytokines. Nat Rev Immunol 9: 480-490, 2009.

6. Migone TS, Lin JX, Cereseto A, Mulloy JC, O'Shea JJ, Franchini $\mathrm{G}$ and Leonard WJ: Constitutively activated Jak-STAT pathway in T cells transformed with HTLV-I. Science 269: 79-81, 1995.

7. Xu X, Kang SH, Heidenreich O, Okerholm M, O'Shea JJ and Nerenberg MI: Constitutive activation of different Jak tyrosine kinases in human T cell leukemia virus type 1 (HTLV-1) tax protein or virus-transformed cells. J Clin Invest 96: 1548-1555, 1995.

8. Takemoto S, Mulloy JC, Cereseto A, Migone T-S, Patel BKR, Matsuoka M, Yamaguchi K, Takatsuki K, Kamihira S, White JD, et al: Proliferation of adult $\mathrm{T}$ cell leukemia/lymphoma cells is associated with the constitutive activation of JAK/STAT proteins. Proc Natl Acad Sci USA 94: 13897-13902, 1997.

9. Ju W, Zhang M, Jiang J-K, Thomas CJ, Oh U, Bryant BR, Chen J, Sato N, Tagaya Y, Morris JC, et al: CP-690,550, a therapeutic agent, inhibits cytokine-mediated Jak3 activation and proliferation of T cells from patients with ATL and HAM/TSP. Blood 117: 1938-1946, 2011.

10. Zhang M, Mathews Griner LA, Ju W, Duveau DY, Guha R, Petrus MN, Wen B, Maeda M, Shinn P, Ferrer M, et al: Selective targeting of JAK/STAT signaling is potentiated by $\mathrm{Bcl}-\mathrm{xL}$ blockade in IL-2-dependent adult T-cell leukemia. Proc Natl Acad Sci USA 112: 12480-12485, 2015.

11. Weil R, Levraud J-P, Dodon MD, Bessia C, Hazan U, Kourilsky P and Israël A: Altered expression of tyrosine kinases of the Src and Syk families in human T-cell leukemia virus type 1-infected T-cell lines. J Virol 73: 3709-3717, 1999.

12. Coffey G, Betz A, DeGuzman F, Pak Y, Inagaki M, Baker DC Hollenbach SJ, Pandey A and Sinha U: The novel kinase inhibitor PRT062070 (Cerdulatinib) demonstrates efficacy in models of autoimmunity and B-cell cancer. J Pharmacol Exp Ther 351: 538-548, 2014

13. Ma J, Xing W, Coffey G, Dresser K, Lu K, Guo A, Raca G, Pandey A, Conley P, Yu H, et al: Cerdulatinib, a novel dual SYK/ JAK kinase inhibitor, has broad anti-tumor activity in both ABC and GCB types of diffuse large B cell lymphoma. Oncotarget 6: 43881-43896, 2015. 
14. Blunt MD, Koehrer S, Dobson RC, Larrayoz M, Wilmore S, Hayman A, Parnell J, Smith LD, Davies A, Johnson PWM, et al: The dual Syk/JAK inhibitor cerdulatinib antagonizes B-cell receptor and microenvironmental signaling in chronic lymphocytic leukemia. Clin Cancer Res 23: 2313-2324, 2017.

15. Guo A, Lu P, Coffey G, Conley P, Pandey A and Wang YL: Dual SYK/JAK inhibition overcomes ibrutinib resistance in chronic lymphocytic leukemia: Cerdulatinib, but not ibrutinib, induces apoptosis of tumor cells protected by the microenvironment. Oncotarget 8: 12953-12967, 2017.

16. Liu D and Mamorska-Dyga A: Syk inhibitors in clinical development for hematological malignancies. J Hematol Oncol 10: 145, 2017.

17. Stapper NJ, Stuschke M, Sak A and Stüben G: Radiation-induced apoptosis in human sarcoma and glioma cell lines. Int J Cancer 62: 58-62, 1995 .

18. Mori N and Prager D: Transactivation of the interleukin-1alpha promoter by human T-cell leukemia virus type I and type II Tax proteins. Blood 87: 3410-3417, 1996.

19. Ishikawa C, Senba M and Mori N: Butein inhibits NF- $x$ B, AP-1 and Akt activation in adult T-cell leukemia/lymphoma. Int J Oncol 51: 633-643, 2017.

20. Tomayko MM and Reynolds CP: Determination of subcutaneous tumor size in athymic (nude) mice. Cancer Chemother Pharmacol 24: $148-154,1989$.

21. Mócsai A, Ruland J and Tybulewicz VL: The SYK tyrosine kinase: A crucial player in diverse biological functions. Nat Rev Immunol 10: 387-402, 2010.

22. Zheng Z, Li Z, Chen S, Pan J and Ma X: Tetramethylpyrazine attenuates TNF- $\alpha$-induced iNOS expression in human endothelial cells: Involvement of Syk-mediated activation of PI3K-IKK-ІкB signaling pathways. Exp Cell Res 319: 2145-2151, 2013.

23. Hatton O, Lambert SL, Krams SM and Martinez OM: Src kinase and Syk activation initiate PI3K signaling by a chimeric latent membrane protein 1 in Epstein-Barr virus (EBV)+ B cell lymphomas. PLoS One 7: e42610, 2012.

24. Wang LH, Kirken RA, Erwin RA, Yu C-R and Farrar WL: JAK3 STAT, and MAPK signaling pathways as novel molecular targets for the tyrphostin AG-490 regulation of IL-2-mediated T cell response. J Immunol 162: 3897-3904, 1999.

25. Sherr CJ: Cancer cell cycles. Science 274: 1672-1677, 1996.

26. Karimian A, Ahmadi Y and Yousefi B: Multiple functions of p21 in cell cycle, apoptosis and transcriptional regulation after DNA damage. DNA Repair (Amst) 42: 63-71, 2016.

27. Hu X and Moscinski LC: Cdc2: A monopotent or pluripotent CDK? Cell Prolif 44: 205-211, 2011.

28. Guadagno TM and Newport JW: Cdk2 kinase is required for entry into mitosis as a positive regulator of $\mathrm{Cdc} 2$-cyclin $\mathrm{B}$ kinase activity. Cell 84: 73-82, 1996.
29. Yadav S, Kalra N, Ganju L and Singh M: Activator protein-1 (AP-1): A bridge between life and death in lung epithelial (A549) cells under hypoxia. Mol Cell Biochem 436: 99-110, 2017.

30. Zhang Y-X, Li X-F, Yuan G-Q, Hu H, Song X-Y, Li J-Y, Miao X-K, Zhou T-X, Yang W-L, Zhang X-W, et al: $\beta$-Arrestin 1 has an essential role in neurokinin-1 receptor-mediated glioblastoma cell proliferation and $\mathrm{G}_{2} / \mathrm{M}$ phase transition. J Biol Chem 292: 8933-8947, 2017.

31. Manning BD and Cantley LC: AKT/PKB signaling: Navigating downstream. Cell 129: 1261-1274, 2007.

32. Roy SK, Srivastava RK and Shankar S: Inhibition of PI3K/ AKT and MAPK/ERK pathways causes activation of FOXO transcription factor, leading to cell cycle arrest and apoptosis in pancreatic cancer. J Mol Signal 5: 10, 2010.

33. Das D, Pintucci G and Stern A: MAPK-dependent expression of p21(WAF) and p27(kip1) in PMA-induced differentiation of HL60 cells. FEBS Lett 472: 50-52, 2000.

34. Kim R, Tanabe K, Uchida Y, Emi M, Inoue H and Toge T: Current status of the molecular mechanisms of anticancer drug-induced apoptosis. The contribution of molecular-level analysis to cancer chemotherapy. Cancer Chemother Pharmacol 50: 343-352, 2002.

35. Krueger A, Baumann S, Krammer PH and Kirchhoff S: FLICEinhibitory proteins: Regulators of death receptor-mediated apoptosis. Mol Cell Biol 21: 8247-8254, 2001.

36. Catlett-Falcone R, Dalton WS and Jove R: STAT proteins as novel targets for cancer therapy. Signal transducer an activator of transcription. Curr Opin Oncol 11: 490-496, 1999.

37. Zheng HC: The molecular mechanisms of chemoresistance in cancers. Oncotarget 8: 59950-59964, 2017.

38. Allan LA, Morrice N, Brady S, Magee G, Pathak S and Clarke PR: Inhibition of caspase-9 through phosphorylation at Thr 125 by ERK MAPK. Nat Cell Biol 5: 647-654, 2003.

39. Asselin E, Mills GB and Tsang BK: XIAP regulates Akt activity and caspase-3-dependent cleavage during cisplatin-induced apoptosis in human ovarian epithelial cancer cells. Cancer Res 61: 1862-1868, 2001

40. Liang YL, Wang LY, Wu H, Ma DZ, Xu Z and Zha XL: PKB phosphorylation and survivin expression are cooperatively regulated by disruption of microfilament cytoskeleton. Mol Cell Biochem 254: 257-263, 2003

41. Vaira V, Lee CW, Goel HL, Bosari S, Languino LR and Altieri DC: Regulation of survivin expression by IGF-1/mTOR signaling. Oncogene 26: 2678-2684, 2007.

42. Hamlin PA, Farber CM, Fenske TS, Khatcheressian JL, Miller CB, Munoz J, Patel MR, Schreeder MT, Smith SM, Stevens DA, et al: The dual SYK/JAK inhibitor cerdulatinib demonstrates rapid tumor responses in a phase 2 study in patients with relapsed/refractory B-cell malignancies. Hematol Oncol 35: $74,2017$. 